Prepared in cooperation with the Federal Emergency Management Agency

\title{
Monitoring Storm Tide, Flooding, and Precipitation From Hurricane Maria in Puerto Rico and the U.S. Virgin Islands, September 2017
}

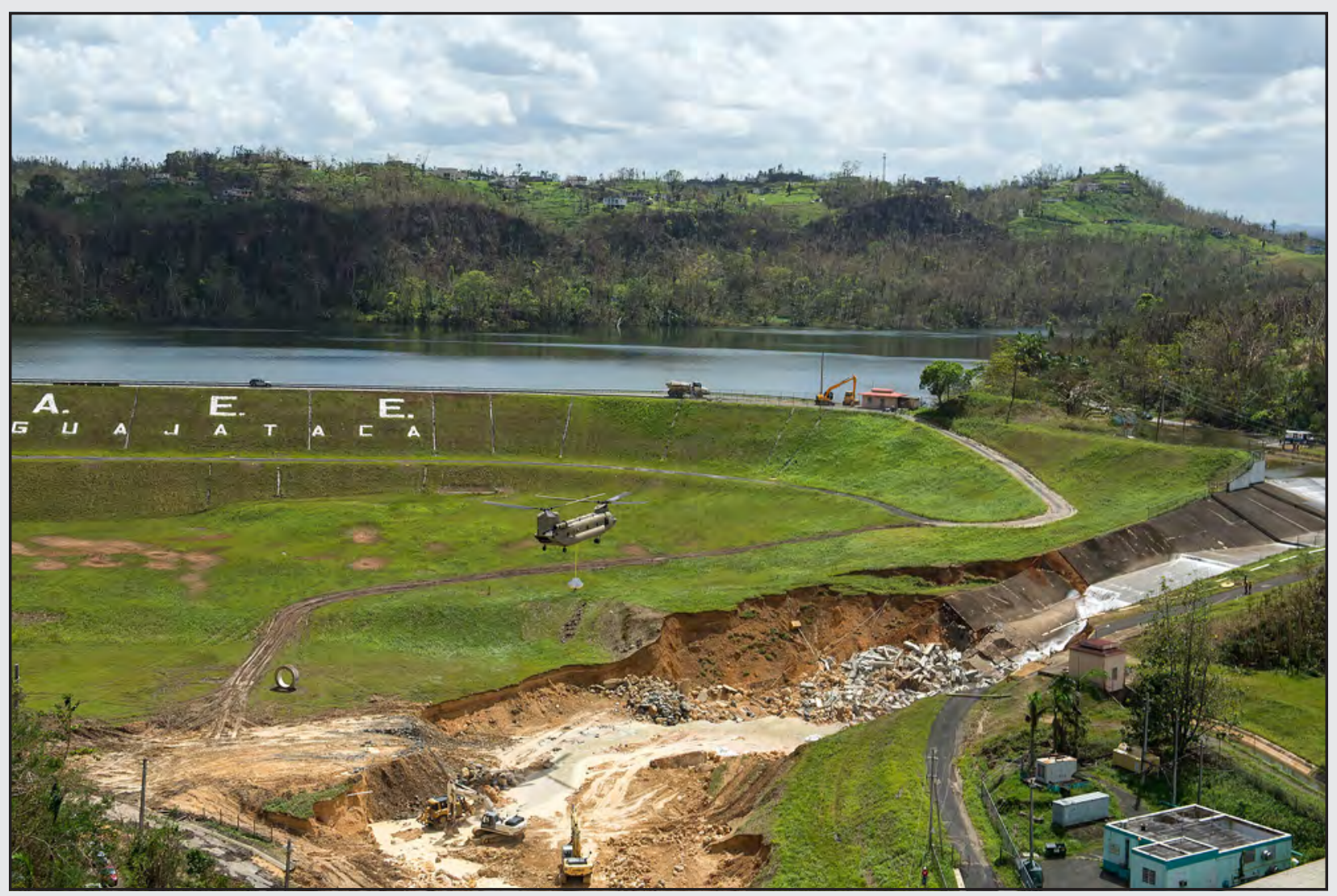

Open-File Report 2019-1065 
Front cover. U.S. Army helicopter installing sandbags to the breached spillway of the Guajataca Dam in Quebradillas, Puerto Rico, October 9, 2017. Photograph courtesy of Staff Sgt. Mark Scovell, U.S. Army.

Back cover. Destruction of boat pier in Patillas Municipality, Puerto Rico, September 18 and October 10, 2017. Storm-surge sensor PAPAT2231 attached to left nearshore piling. Photographs by David Rivera, U.S. Geological Survey 


\section{Monitoring Storm Tide, Flooding, and Precipitation From Hurricane Maria in Puerto Rico and the U.S. Virgin Islands, September 2017}

By Michael J. Byrne, Sr.

Prepared in cooperation with the Federal Emergency Management Agency

Open-File Report 2019-1065 


\title{
U.S. Department of the Interior DAVID BERNHARDT, Secretary
}

\author{
U.S. Geological Survey \\ James F. Reilly II, Director
}

U.S. Geological Survey, Reston, Virginia: 2019

For more information on the USGS - the Federal source for science about the Earth, its natural and living resources, natural hazards, and the environment-visit https://www.usgs.gov or call 1-888-ASK-USGS.

For an overview of USGS information products, including maps, imagery, and publications, visit https://store.usgs.gov.

Any use of trade, firm, or product names is for descriptive purposes only and does not imply endorsement by the U.S. Government.

Although this information product, for the most part, is in the public domain, it also may contain copyrighted materials as noted in the text. Permission to reproduce copyrighted items must be secured from the copyright owner.

Suggested citation:

Byrne, M.J., Sr., 2019, Monitoring storm tide, flooding, and precipitation from Hurricane Maria in Puerto Rico and the U.S. Virgin Islands, September 2017: U.S. Geological Survey Open-File Report 2019-1065,16 p., https://doi.org/10.3133/ofr20191065.

ISSN 0196-1497 (print)

ISSN 2331-1258 (online) 


\section{Acknowledgments}

This mission could only be accomplished with U.S. Geological Survey personnel in Puerto Rico and the continental United States and the help of U.S. Military, first responders and other Federal agency partners. 



\section{Contents}

Abstract

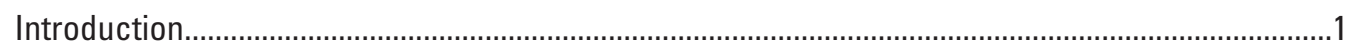

Hurricane Maria Storm Tide, Flood Monitoring, and Precipitation .................................................

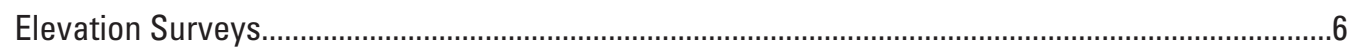

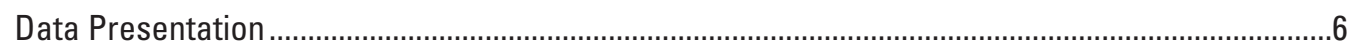

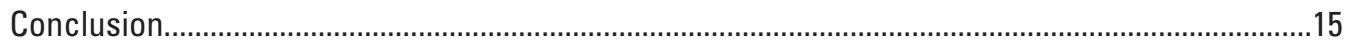

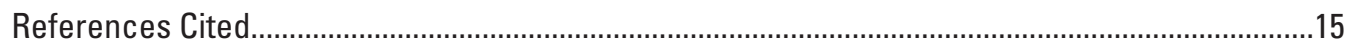

\section{Figures}

1. Map showing Hurricane Maria path and intensity, September 2017 ..............................2

2. Map showing location of water-level sensors for monitoring the timing, areal extent, and magnitude of storm tide and coastal flooding generated by

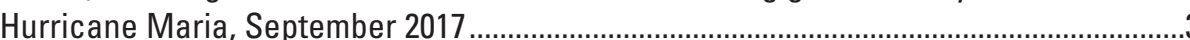

3. Photograph showing U.S. Geological Survey technicians Belytza Velez-Gamez and Dennis Rivera surveying a water-level sensor in the Arroyo Municipality, Puerto Rico, September 2017..

4. Screenshot of hydrograph from the U.S. Geological Survey Flood Event Viewer showing storm-tide elevation and barometric pressure data recorded during Hurricane Maria in Puerto Maunabo, September 2017

5. Photograph showing Puerto Rico National Guard patrolling a main highway in downtown San Juan on September 22, 2017, two days after Hurricane Maria.................6

6. Photograph showing Global Navigation Satellite System equipment used for elevation survey near Puerto Chico, Fajarado, Puerto Rico, October 2017.

\section{Tables}

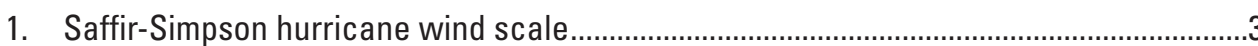

2. Hurricane Maria peak storm-tide data for storm-tide stations in Puerto Rico, September 2017

3. Hurricane Maria high-water marks recorded by the U.S. Geological Survey in Puerto Rico, September 2017

4. Hurricane Maria peak riverine stage recorded at U.S. Geological Survey long-term monitoring sites in Puerto Rico, September 2017

5. Hurricane Maria peak storm-tide data recorded at National Oceanic and Atmospheric Administration real-time tide-gages in Puerto Rico and the U.S. Virgin Islands, September 2017

6. Precipitation measured in the U.S. Virgin Islands and Puerto Rico, September 18-22, 2017. 


\section{Conversion Factors}

U.S. customary units to International System of Units

\begin{tabular}{|c|c|c|}
\hline Multiply & By & To obtain \\
\hline \multicolumn{3}{|c|}{ Length } \\
\hline foot $(\mathrm{ft})$ & 0.3048 & meter $(\mathrm{m})$ \\
\hline mile (mi) & 1.609 & kilometer $(\mathrm{km})$ \\
\hline \multicolumn{3}{|c|}{ Velocity } \\
\hline mile per hour (mi/h) & 1.609 & kilometer per hour $(\mathrm{km} / \mathrm{h})$ \\
\hline
\end{tabular}

\section{Datum}

Vertical coordinate information is referenced to the Puerto Rico Vertical Datum of 2002 (PRVD02), unless otherwise noted.

Horizontal coordinate information is referenced to the North American Datum of 1983 (NAD 83). Elevation, as used in this report, refers to distance above the vertical datum.

\section{Abbreviations}

ASCII

GMT

American Standard Code for Information Interchange

GNSS

Greenwich Mean Time

HWM

Global Navigation Satellite Systems

NetCDF

high-water mark

NGS

Network Common Data Form

NOAA

National Geodetic Survey

NWIS

National Oceanic and Atmospheric Administration

USGS

National Water Information System

U.S. Geological Survey 


\title{
Monitoring Storm Tide, Flooding, and Precipitation From Hurricane Maria in Puerto Rico and the U.S. Virgin Islands, September 2017
}

\author{
By Michael J. Byrne, Sr.
}

\begin{abstract}
Hurricane Maria made landfall south of Yabucoa Harbor, Puerto Rico, as a category 4 hurricane with maximum sustained winds of 155 miles per hour on September 20, 2017. The hurricane devastated much of the U.S. Virgin Islands and Puerto Rico. The U.S. Geological Survey (USGS), in cooperation with Federal Emergency Management Agency, installed a temporary monitoring network of 13 water-level and barometric pressure sensors along the coast of Puerto Rico prior to the storm. In addition to the temporary sensors, the USGS maintains 99 permanent real-time streamgages and 36 real-time precipitation stations in Puerto Rico. The real-time data, updated hourly, during and after the hurricane are displayed in the USGS Flood Event Viewer (https://stn.wim.usgs.gov/FEV/\#MariaSeptember2017) and in the USGS National Water Information System.

The USGS measured 181 coastal and riverine high-water marks throughout Puerto Rico after the storm. Water elevations are referenced to the Puerto Rico Vertical Datum of 2002 (PRVD02) and local datums in Puerto Rico and to the U.S. Virgin Islands Vertical Datum of 2009 (VIVD09) in the U.S. Virgin Islands. Data from the Hurricane Maria storm-tide network are available in tab-delimited, American Standard Code for Information Interchange (ASCII) format and Network Common Data Form (NetCDF) format by site for each sensor through the USGS Flood Event Viewer.
\end{abstract}

\section{Introduction}

Hurricane Maria made landfall south of Yabucoa Harbor, Puerto Rico (fig. 1) as a category 4 hurricane on the Saffir-Simpson Hurricane Wind Scale (table 1) with maximum sustained winds of 155 miles per hour $(\mathrm{mi} / \mathrm{h})$ on September 20, 2017 (Pasch and others, 2018). The hurricane passed over the mountains, weakened, and exited the island the same day near the Isabela Municipality as a category 3 hurricane with maximum sustained winds of $115 \mathrm{mi} / \mathrm{h}$. Puerto Rico and the U.S. Virgin Islands (St. Thomas, St. Croix, and St. John) sustained catastrophic damage because of the high winds, storm tide, flooding, and precipitation. Prior to Hurricane Maria landfall, Hurricane Irma skirted the coast of the islands on September 7, 2017, as a category 5 hurricane with maximum sustained winds of $185 \mathrm{mi} / \mathrm{h}$ (Cangialosi and others, 2018), causing 55 percent of the 3.3 million people living in Puerto Rico (U.S. Census Bureau, 2017) and 100 percent of the 106,000 people living in the U.S. Virgin Islands (U.S. Census Bureau, 2010) to lose electrical power (U.S. Department of Energy, 2017). Hurricane Maria devastated much of the remaining electrical grid, leaving most of the islands without electricity. As of February 2018, power had been restored to 70 percent of Puerto Rico and 100 percent of the U.S. Virgin Islands (U.S. Department of Energy, 2018). On August 14, 2018, the Puerto Rico Electric Power Authority announced power was restored to 100 percent of Puerto Rico (Autoridad de Energía Eléctrica, 2018).

In advance of landfall of Hurricane Maria, the U.S. Geological Survey (USGS), in cooperation with the Federal Emergency Management Agency, deployed a temporary monitoring network of water-level sensors and barometric pressure sensors at 13 locations along the Puerto Rico coast to record the timing, areal extent, and magnitude of hurricane-generated storm tide and coastal flooding (fig. 2). The deployment of Hurricane Maria water-level sensors was completed as part of a coordinated Federal emergency response as outlined by the Robert T. Stafford Disaster Relief and Emergency Assistance Act (42 U.S.C. 5121 et seq.) under a directed mission assignment by the Federal Emergency Management Agency. Storm tide is the water-level rise above the astronomical tide during a coastal storm. As noted by Frantz and others (2017), these data are used to improve the performance of storm-tide models used to predict the maximum and incremental water levels and flood extent; these models also predict the site-specific effects of storm tide and flooding on natural and anthropogenic features of the environment.

The permanent USGS monitoring network of real-time streamgages, precipitation stations, and National Oceanic and Atmospheric Administration (NOAA) tide-gages in Puerto Rico and the U.S. Virgin Islands relayed data hourly for display on the USGS Flood Event Viewer (https://stn.wim.usgs.gov/FEV/\#MariaSeptember2017) and 


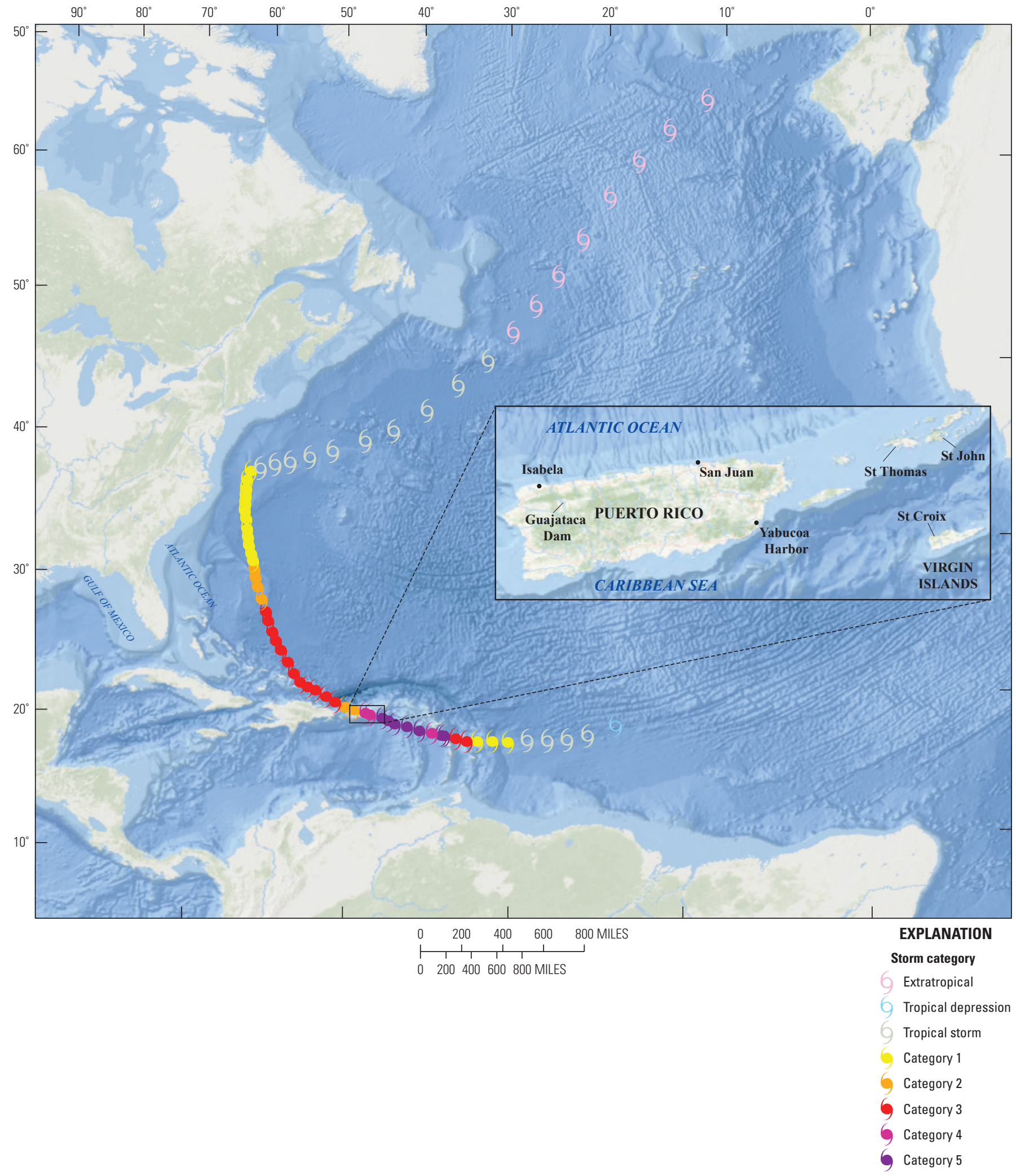

Figure 1. Hurricane Maria path and intensity, September 2017. Map image is the intellectual property of Esri and is used herein under license. Copyright (c) 2017 Esri and its licensors. All rights reserved. 
Table 1. Saffir-Simpson hurricane wind scale.

[National Hurricane Center (2012). mi/h, mile per hour; >, greater than]

\begin{tabular}{ccl}
\hline Category & $\begin{array}{c}\text { Wind } \\
\text { speed } \\
\text { (mi/h) }\end{array}$ & \\
\hline 1 & $74-95$ & Very dangerous wind will produce some damage. \\
2 & $96-110$ & Extremely dangerous winds will cause extensive damage. \\
3 & $111-129$ & Devastating damage will occur. \\
4 & $130-156$ & Catastrophic damage will occur. \\
5 & $>157$ & Catastrophic damage will occur. \\
\hline
\end{tabular}

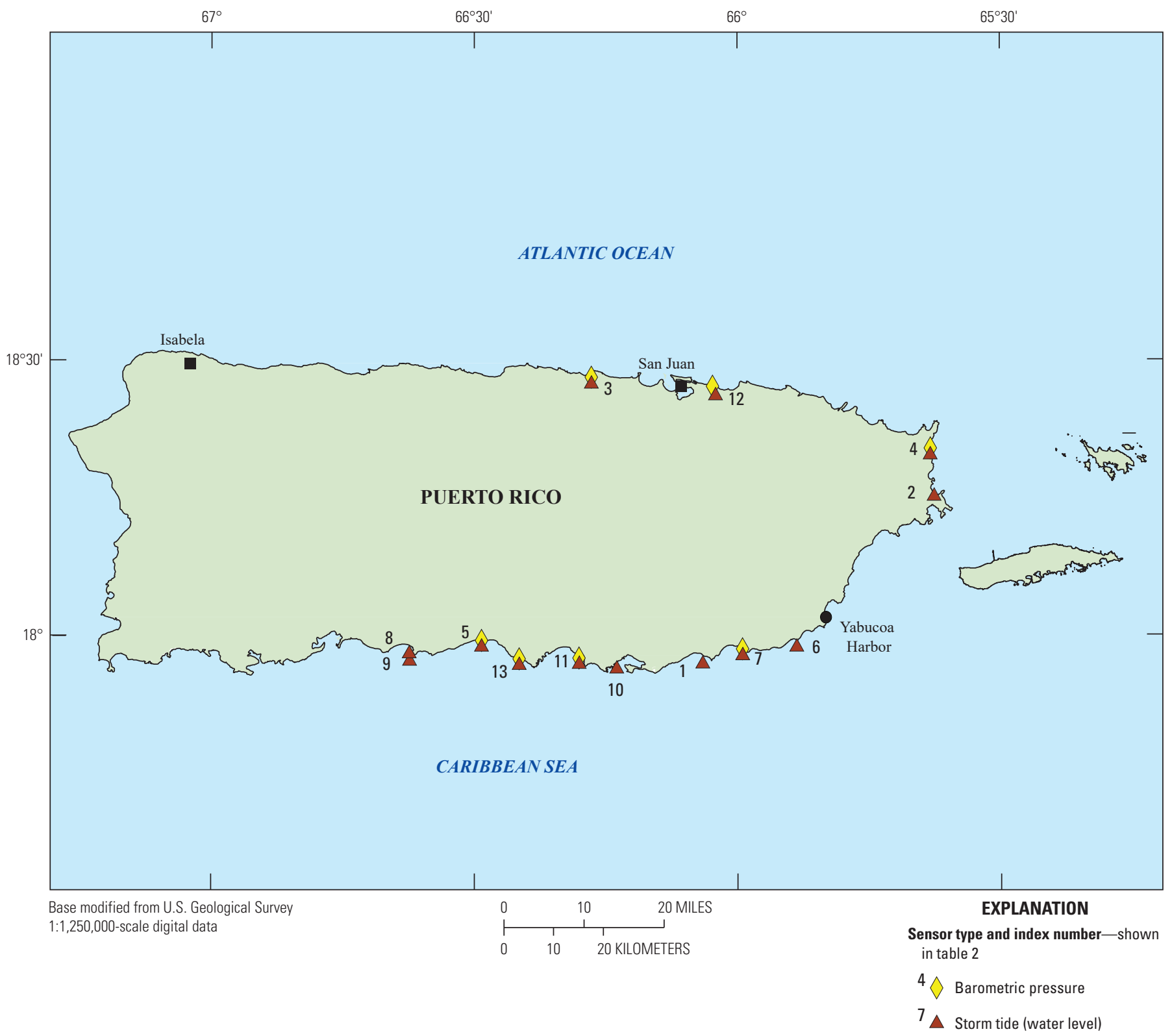

Figure 2. Location of water-level sensors for monitoring the timing, areal extent, and magnitude of storm tide and coastal flooding generated by Hurricane Maria, September 2017. 
in the National Water Information System (NWIS; U.S. Geological Survey, 2016; NOAA, 2017). These real-time data provided emergency managers and responders with critical information for identifying flood-affected areas and accurately directing assistance to affected communities.

\section{Hurricane Maria Storm Tide, Flood Monitoring, and Precipitation}

Water-level sensors were placed at sites in Puerto Rico selected to augment existing tide-gage networks and to ensure adequate monitoring in areas forecast to have substantial storm tide. A total of 13 water-level sensors and seven barometric pressure sensors were deployed at 13 sites on September 18, 2017, two days before hurricane landfall (fig. 2, table 2). The water-level and barometric pressure sensors were programmed to record atmospheric pressure data, in pounds per square inch, at 30 -second intervals beginning at 0600 Greenwich Mean Time (GMT) on September 19 through 1800 GMT on September 26. Two water-level sensors were destroyed during the storm. A typical sensor installation is shown in figure 3 .

The water-level and barometric pressure sensor data were collected and processed following protocols established by McGee and others (2006) and McCallum and others (2012). In order to compute the storm-tide time-series datasets in feet above the Puerto Rico Vertical Datum of 2002 (PRVD02) at each site, a post-processing script was used to correct the water-level sensor data for barometric pressure and salinity, and to adjust the data to the elevation of the respective reference point. The script also filtered the water-level data to determine the peak storm tide using the method described in Frantz and others (2017). An example of the water-level data, barometric pressure data, and selected maximum storm-tide elevation are shown in figure 4.

Several permanent USGS real-time streamgages and precipitation stations were destroyed or malfunctioned because of the hurricane-force winds and flooding associated with Hurricane Irma and Hurricane Maria. High flows associated with the hurricanes potentially altered the channel geometry of many rivers, thereby altering the hydraulic rating curves used to estimate river discharge. The USGS conducted direct

Table 2. Hurricane Maria peak storm-tide data for storm-tide stations in Puerto Rico, September 2017.

[Dates shown as month/day/year. Time shown in 24-hour format. PRVD02, Puerto Rico Vertical Datum 2002; UTC, Coordinated Universal Time; \pm , plus or minus; ft, foot; --, not applicable; n.d., no data (sensor destroyed during storm)]

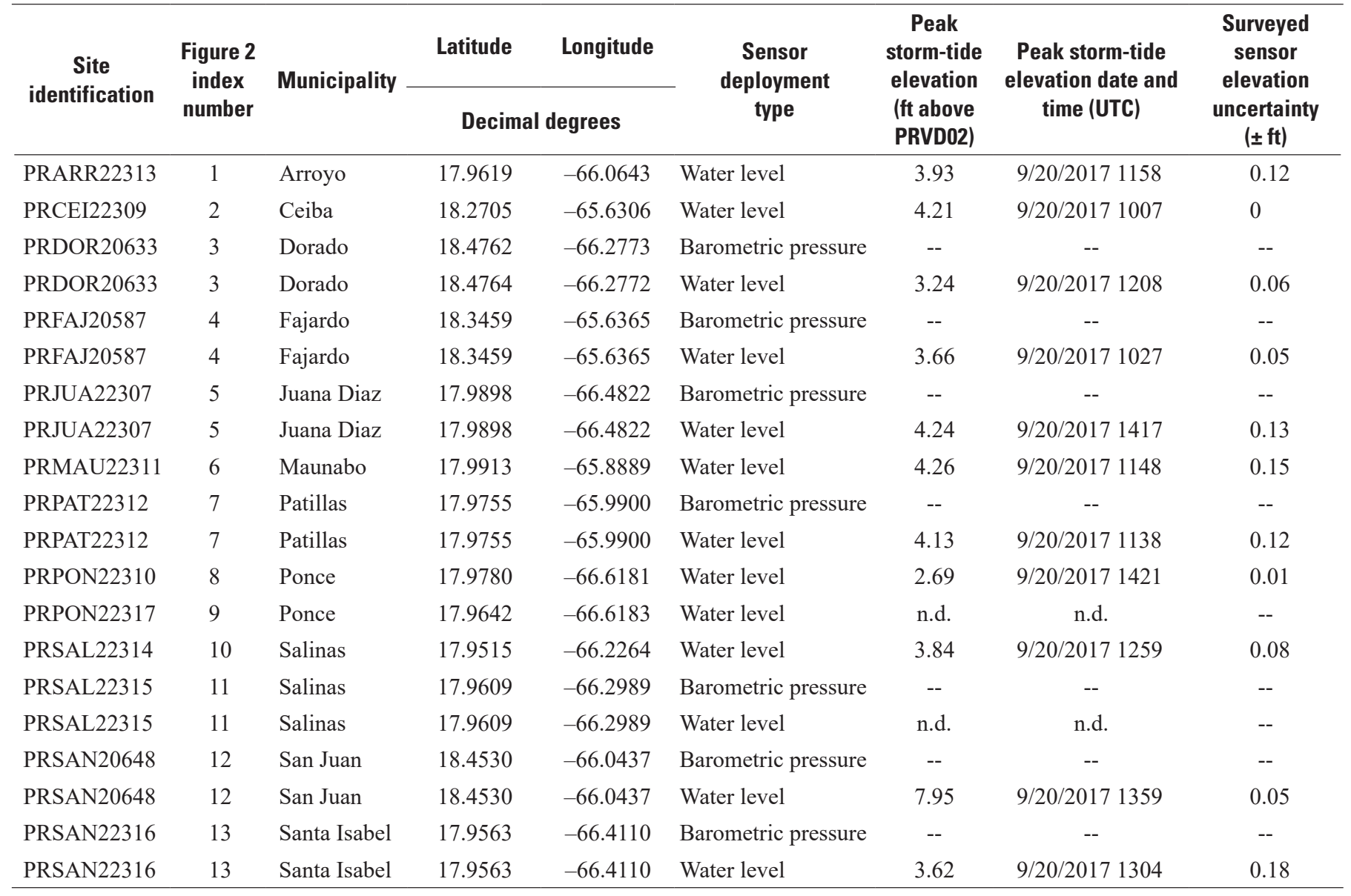




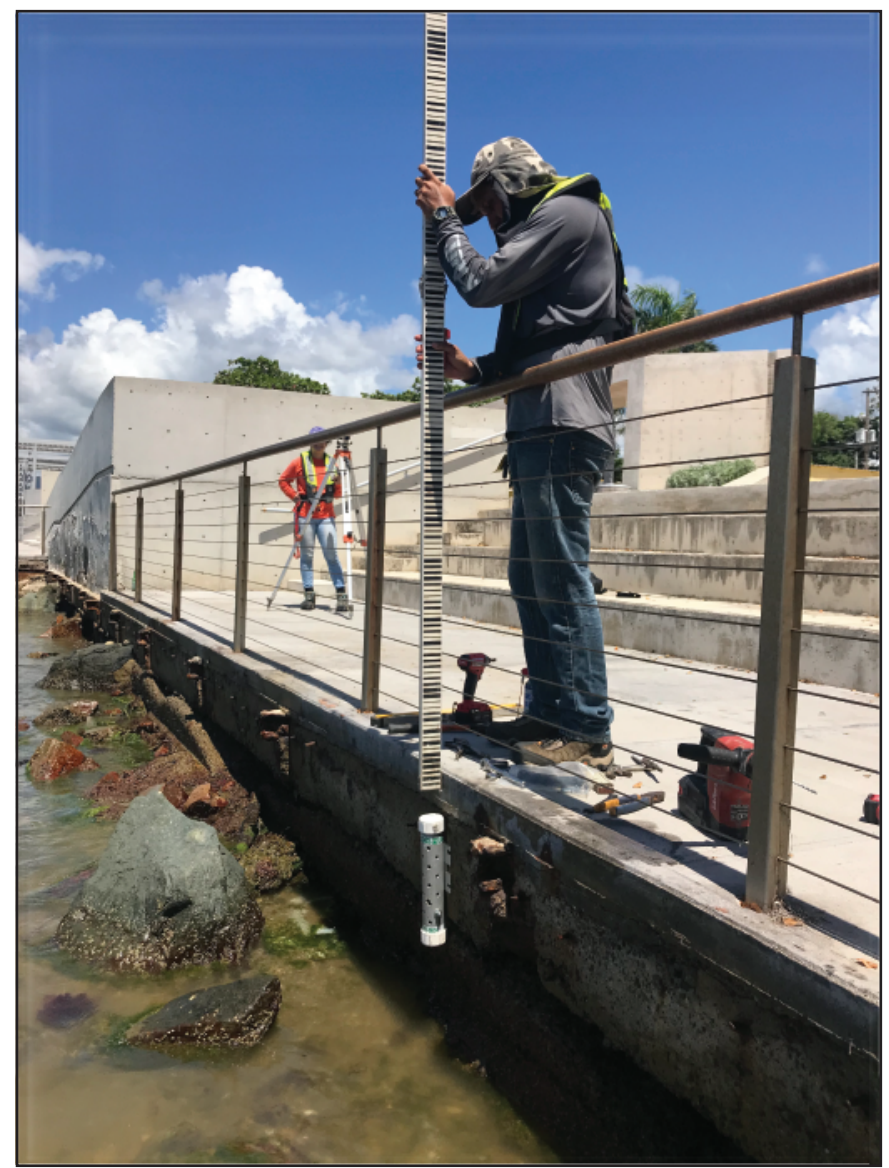

Figure 3. U.S. Geological Survey (USGS) technicians Belytza Velez-Gamez and Dennis Rivera surveying a water-level sensor in the Arroyo Municipality, Puerto Rico, September 2017. Photograph by James Torres, USGS.

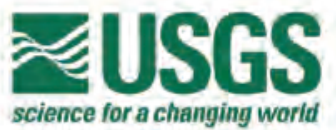

EXPLANATION

- Unfittered Water Elevation

- Stom nde (Lowpass filtered) water Elonation

Recordabie Water Elevation

- Maximum Unfitered Water Elevation

A Maximum Starm Tide water Elevation

Storm Tide Water Elevation, Latitude: 17.9913 Longitude: -65.8889 STN Site ID: PRMAU22311

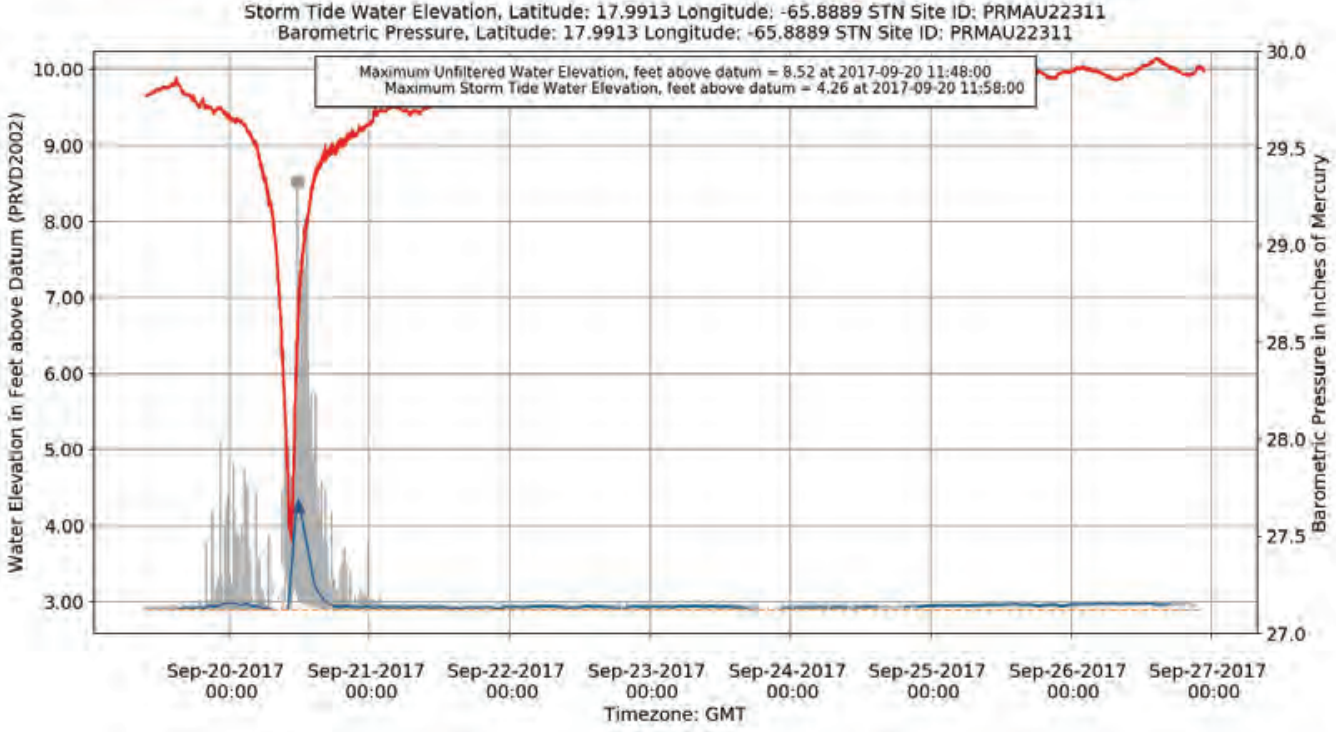

Figure 4. Hydrograph from the U.S. Geological Survey Flood Event Viewer (https://stn. wim.usgs.gov/FEV/\#MariaSeptember2017) showing storm-tide elevation and barometric pressure data recorded during Hurricane Maria in Puerto Maunabo, September 2017. 
discharge measurements, indirect discharge measurements (Matthai, 1967), and step backwater analyses (Davidian, 1976) in Puerto Rico in the months following the hurricane to guide development of new rating curves appropriate to post-hurricane hydraulic conditions. The top priorities of the USGS, following the hurricane, were retrieving the water-level sensors, repairing the permanent gages, making discharge measurements, and flagging high-water marks. Progress to repair and rebuild the sites was hampered by flooded roadways, fuel shortages, collapsed bridges, shortage of construction material, equipment, power outages, and mudslides (fig. 5). Assistance from USGS personnel in the contiguous United States was delayed more than a week after the hurricane because of limited commercial flights (Federal Aviation Administration, 2017).

In total, 181 coastal and riverine high-water marks (HWMs) located in Puerto Rico were recovered and surveyed following the techniques described in Koenig and others (2016). The high-water mark and storm-tide sensor data were disseminated on the USGS Flood Event Viewer website (https://stn.wim.usgs.gov/FEV/\#MariaSeptember2017).

\section{Elevation Surveys}

National Geodetic Survey (NGS) benchmarks were used to determine vertical control at permanent and storm-tide monitoring stations throughout the study area. At the majority of the sites, survey-grade Global Navigation Satellite

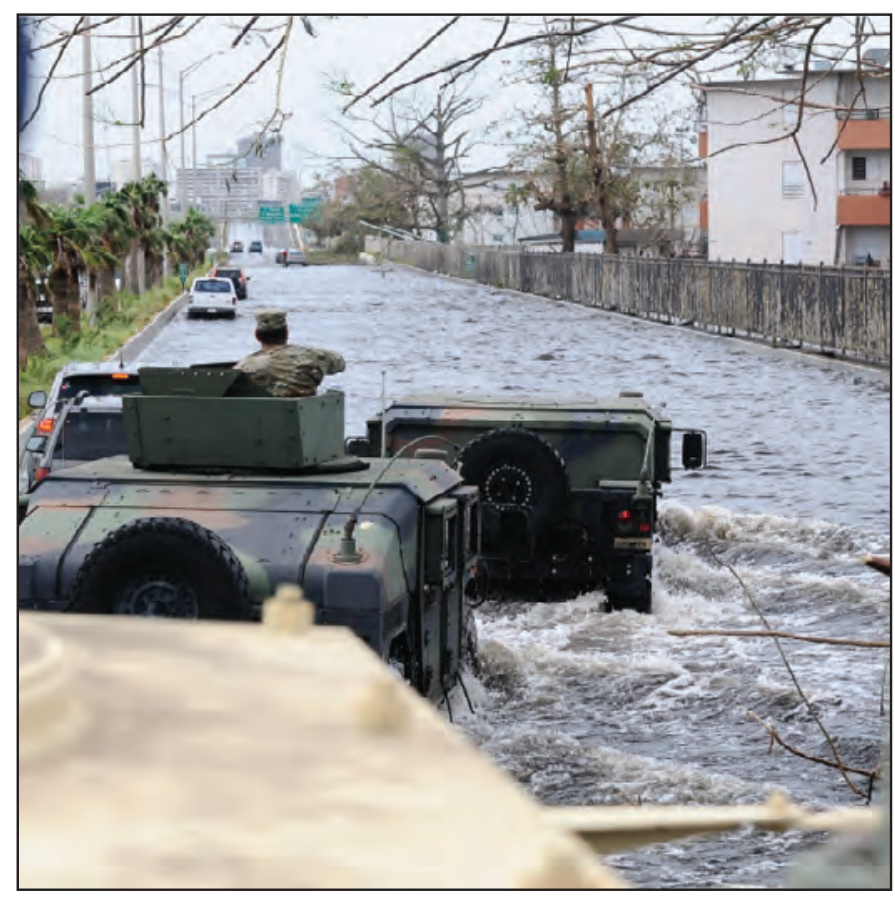

Figure 5. Puerto Rico National Guard patrolling a main highway in downtown San Juan on September 22, 2017, two days after Hurricane Maria. Photograph courtesy of Sgt. Jose Ahiram DiazRamos, U.S. National Guard.
System (GNSS) equipment (fig. 6) was used to determine the elevation above PRVD02 of the reference points and HWMs, in accordance with USGS technical guidance (Rydlund and Densmore, 2012); all GNSS-determined elevations in this report were derived using the GEOID12A model (NGS, 2017). Vertical control was established on permanent objects near the stations to convert the recorded water-surface elevations to the PRVD02 datum. The survey uncertainty was calculated using the methods described in Rydlund and Densmore (2012). At the storm-tide stations, graduated steel tapes were used to relate the elevations of the reference points to those of the water-level sensors.

\section{Data Presentation}

The data from the Hurricane Maria storm-tide network are available in tab-delimited, American Standard Code for Information Interchange (ASCII) format and Network Common Data Form (NetCDF) format by site for each sensor from the USGS Flood Event Viewer (https://stn.wim.usgs. gov/FEV/\#MariaSeptember2017). Digital photographs for selected locations are available on the viewer. Data for each sensor include location, date, time, water level, and barometric pressure. Data for HWMs include location, description and quality of the mark, and elevation.

The peak storm tide was recorded at 11 water-level sensors (table 2). All HWM data collected by the USGS immediately after Hurricane Maria are listed in table 3. The survey uncertainty for the water-level sensors and high-water mark elevations is reported in tables 2 and 3, respectively. The peak riverine stage was recorded at 70 permanent stations (table 4); 24 canal and lake station peaks were excluded from this report. Some data are considered provisional, as denoted by an asterisk, until they receive a formal review by the USGS. Peak storm-tide data also were compiled for 8 of 13 functioning NOAA tide-gages, which have instrumentation to measure coastal water levels located in Puerto Rico and the U.S. Virgin Islands (table 5; NOAA, 2017). Precipitation was recorded at 17 permanent stations (table 6).

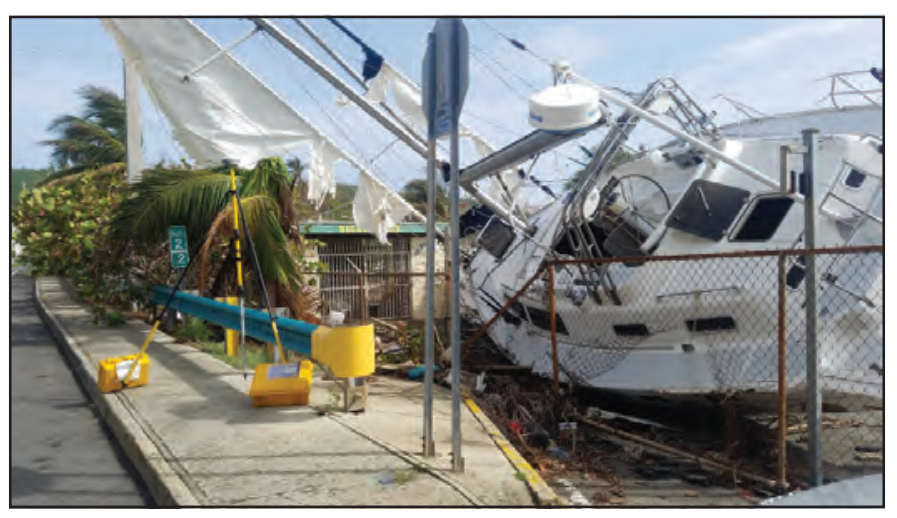

Figure 6. Global Navigation Satellite System equipment used for elevation survey near Puerto Chico, Fajarado, Puerto Rico, October 2017. Photograph by Ryan Hollins, U.S. Geological Survey. 
Table 3. Hurricane Maria high-water marks recorded by the U.S. Geological Survey in Puerto Rico, September 2017.

[Peak assumed to occur on September 20, 2017. HWM, high-water mark; ft, foot; PRVD02, Puerto Rico Vertical Datum of $2002 ; \pm$, plus or minus]

\begin{tabular}{|c|c|c|c|c|c|}
\hline \multirow{2}{*}{$\begin{array}{c}\text { Site } \\
\text { identification }\end{array}$} & \multirow{2}{*}{ Municipality } & Latitude & Longitude & \multirow{2}{*}{$\begin{array}{c}\text { HWM } \\
\text { surveyed elevation } \\
\text { (ft above PRVD02) }\end{array}$} & \multirow{2}{*}{$\begin{array}{l}\text { HWM survey } \\
\text { uncertainty } \\
( \pm \mathrm{ft})\end{array}$} \\
\hline & & \multicolumn{2}{|c|}{ Decimal degrees } & & \\
\hline PRPAT22312 & Patillas & 17.9755 & -65.9900 & 4.62 & 0.12 \\
\hline PRPAT22312 & Patillas & 17.9755 & -65.9900 & 4.99 & 0.12 \\
\hline PRCEI22309 & Ceiba & 18.2705 & -65.6306 & 5.23 & 0.12 \\
\hline PRFAJ20587 & Fajardo & 18.3459 & -65.6365 & 5.51 & 0.14 \\
\hline PRDOR20633 & Dorado & 18.4762 & -66.2773 & 7.44 & 0.06 \\
\hline PRDOR20633 & Dorado & 18.4764 & -66.2772 & 7.03 & 0.06 \\
\hline PRJUA22307 & Juana Diaz & 17.9898 & -66.4822 & 4.74 & 0.13 \\
\hline PRPON22310 & Ponce & 17.9780 & -66.6181 & 5.06 & 0.01 \\
\hline PRSAL22315 & Salinas & 17.9609 & -66.2989 & 5.18 & 0.09 \\
\hline PRYAB20635 & Yabucoa & 18.0639 & -65.8163 & 8.70 & 0.13 \\
\hline PRYAB20635 & Yabucoa & 18.0640 & -65.8165 & 8.37 & 0.13 \\
\hline PRHUM20650 & Humacao & 18.1644 & -65.7439 & 5.93 & 0.13 \\
\hline PRSAN22316 & Santa Isabel & 17.9563 & -66.4110 & 5.19 & 0.18 \\
\hline PRCOR23569 & Corozal & 18.3417 & -66.3212 & 257.60 & 0.08 \\
\hline PRCOR23571 & Corozal & 18.3420 & -66.3198 & 260.93 & 0.08 \\
\hline PRGUR23573 & Gurabo & 18.2571 & -65.9733 & 161.91 & 0.03 \\
\hline PRGUR23574 & Gurabo & 18.2570 & -65.9712 & 162.28 & 0.03 \\
\hline PRTOA23575 & Toa Baja & 18.4437 & -66.2314 & 14.15 & 0.03 \\
\hline PRTOA23577 & Toa Baja & 18.4463 & -66.2437 & 13.81 & 0.03 \\
\hline PRTOA23578 & Toa Baja & 18.4431 & -66.2515 & 17.37 & 0.03 \\
\hline PRTOA23579 & Toa Baja & 18.4398 & -66.2531 & 23.41 & 0.03 \\
\hline PRCAR23570 & Carolina & 18.3800 & -65.9558 & 35.19 & 0.01 \\
\hline PRCOR23598 & Corozal & 18.3419 & -66.3216 & 257.65 & 0.08 \\
\hline PRGUR23572 & Gurabo & 18.2592 & -65.9733 & 162.17 & 0.03 \\
\hline PRTOA23576 & Toa Baja & 18.4460 & -66.2391 & 13.74 & 0.03 \\
\hline PRTOA23599 & Toa Baja & 18.4438 & -66.1784 & 9.56 & 0.11 \\
\hline PRTOA23600 & Toa Baja & 18.4451 & -66.1814 & 9.49 & 0.11 \\
\hline PRTOA23601 & Toa Baja & 18.4471 & -66.1838 & 8.72 & 0.11 \\
\hline PRTOA23602 & Toa Baja & 18.4501 & -66.1860 & 9.62 & 0.11 \\
\hline PRCAR23603 & Carolina & 18.3786 & -65.9473 & 33.77 & 0.01 \\
\hline PRCAR23604 & Carolina & 18.4003 & -65.9458 & 30.91 & 0.01 \\
\hline PRCAR23605 & Carolina & 18.3936 & -65.9306 & 29.58 & 0.01 \\
\hline PRCAR23606 & Carolina & 18.3876 & -65.9205 & 31.72 & 0.01 \\
\hline PRCAR23607 & Carolina & 18.3785 & -65.9531 & 36.95 & 0.01 \\
\hline PRCAR23607 & Carolina & 18.3785 & -65.9531 & 36.76 & 0.01 \\
\hline PRCOR23608 & Corozal & 18.3338 & -66.3144 & 298.01 & 0.08 \\
\hline PRCOR23609 & Corozal & 18.3392 & -66.3169 & 267.27 & 0.08 \\
\hline PRCOR23610 & Corozal & 18.3383 & -66.3194 & 265.29 & 0.08 \\
\hline PRGUR23611 & Gurabo & 18.2631 & -65.9668 & 144.34 & 0.03 \\
\hline
\end{tabular}


Table 3. Hurricane Maria high-water marks recorded by the U.S. Geological Survey in Puerto Rico, September 2017.-Continued

[Peak assumed to occur on September 20, 2017. HWM, high-water mark; ft, foot; PRVD02, Puerto Rico Vertical Datum of $2002 ; \pm$, plus or minus]

\begin{tabular}{|c|c|c|c|c|c|}
\hline \multirow{2}{*}{$\begin{array}{c}\text { Site } \\
\text { identification }\end{array}$} & \multirow{2}{*}{ Municipality } & Latitude & Longitude & \multirow{2}{*}{$\begin{array}{c}\text { HWM } \\
\text { surveyed elevation } \\
\text { (ft above PRVD02) }\end{array}$} & \multirow{2}{*}{$\begin{array}{c}\text { HWM survey } \\
\text { uncertainty } \\
( \pm \mathrm{ft})\end{array}$} \\
\hline & & \multicolumn{2}{|c|}{ Decimal degrees } & & \\
\hline PRTOA23612 & Toa Baja & 18.4537 & -66.1879 & 9.82 & 0.11 \\
\hline PRTOA23613 & Toa Baja & 18.4515 & -66.1911 & 9.94 & 0.11 \\
\hline PRARE23614 & Arecibo & 18.4633 & -66.7220 & 16.89 & 0.07 \\
\hline PRARE23615 & Arecibo & 18.4636 & -66.7237 & 16.86 & 0.07 \\
\hline PRMAN23618 & Manati & 18.4671 & -66.5256 & 17.47 & 0.10 \\
\hline PRMAN23619 & Manati & 18.4476 & -66.5264 & 21.47 & 0.10 \\
\hline PRBAR23620 & Barceloneta & 18.4798 & -66.5353 & 5.72 & 0.10 \\
\hline PRBAR23620 & Barceloneta & 18.4798 & -66.5353 & 5.77 & 0.10 \\
\hline PRSAN23621 & San Juan & 18.4120 & -66.0744 & 16.02 & 0.05 \\
\hline PRBAR23622 & Barceloneta & 18.4648 & -66.5319 & 17.03 & 0.10 \\
\hline PRBAR23622 & Barceloneta & 18.4648 & -66.5319 & 16.96 & 0.10 \\
\hline PRCOM23624 & Comerio & 18.2173 & -66.2273 & 655.83 & 0.16 \\
\hline PRCOM23625 & Comerio & 18.2157 & -66.2300 & 658.15 & 0.16 \\
\hline PRCOM23626 & Comerio & 18.2150 & -66.2276 & 655.89 & 0.16 \\
\hline PRCOM23627 & Comerio & 18.2155 & -66.2255 & 652.26 & 0.16 \\
\hline PRCAN23628 & Canovanas & 18.3886 & -65.9225 & 24.98 & 0.01 \\
\hline PRCAN23629 & Canovanas & 18.3880 & -65.9213 & 30.40 & 0.01 \\
\hline PRCAN23630 & Canovanas & 18.3713 & -65.8973 & 52.53 & 0.05 \\
\hline PRCAN23631 & Canovanas & 18.3811 & -65.8999 & 26.00 & 0.05 \\
\hline PRDOR23632 & Dorado & 18.4565 & -66.2595 & 15.74 & 0.08 \\
\hline PRDOR23633 & Dorado & 18.4567 & -66.2647 & 16.86 & 0.08 \\
\hline PRDOR23634 & Dorado & 18.4588 & -66.2699 & 15.55 & 0.08 \\
\hline PRDOR23635 & Dorado & 18.4328 & -66.2745 & 29.38 & 0.08 \\
\hline PRMAN23623 & Manati & 18.4261 & -66.4963 & 43.69 & 0.10 \\
\hline PRDOR23636 & Dorado & 18.4293 & -66.2653 & 29.47 & 0.08 \\
\hline PRBAR23637 & Barceloneta & 18.4792 & -66.5373 & 9.64 & 0.10 \\
\hline PRBAR23638 & Barceloneta & 18.4710 & -66.5316 & 15.48 & 0.10 \\
\hline PRBAR23638 & Barceloneta & 18.4710 & -66.5316 & 15.34 & 0.10 \\
\hline PRUTU23639 & Utuado & 18.2666 & -66.6978 & 462.78 & 0.06 \\
\hline PRUTU23640 & Utuado & 18.2529 & -66.6776 & 597.31 & 0.06 \\
\hline PRARE23641 & Arecibo & 18.4103 & -66.0758 & 15.59 & 0.00 \\
\hline PRUTU23640 & Utuado & 18.2528 & -66.6777 & 597.79 & 0.06 \\
\hline PRSAN23642 & San Juan & 18.4020 & -66.0659 & 28.42 & 0.00 \\
\hline PRSAL23473 & Salinas & 17.9559 & -66.2189 & 2.12 & 0.09 \\
\hline PRSAL23473 & Salinas & 17.9559 & -66.2189 & 1.90 & 0.09 \\
\hline PRUTU23643 & Utuado & 18.2671 & -66.7030 & 449.08 & 0.06 \\
\hline PRMAN23644 & Manati & 18.4248 & -66.4945 & 46.81 & 0.10 \\
\hline PRMAN23646 & Manati & 18.4249 & -66.4947 & 46.29 & 0.10 \\
\hline PRUTU23645 & Utuado & 18.2667 & -66.7068 & 443.64 & 0.06 \\
\hline
\end{tabular}


Table 3. Hurricane Maria high-water marks recorded by the U.S. Geological Survey in Puerto Rico, September 2017.-Continued

[Peak assumed to occur on September 20, 2017. HWM, high-water mark; ft, foot; PRVD02, Puerto Rico Vertical Datum of $2002 ; \pm$, plus or minus]

\begin{tabular}{|c|c|c|c|c|c|}
\hline \multirow{2}{*}{$\begin{array}{c}\text { Site } \\
\text { identification }\end{array}$} & \multirow{2}{*}{ Municipality } & Latitude & Longitude & \multirow{2}{*}{$\begin{array}{c}\text { HWM } \\
\text { surveyed elevation } \\
\text { (ft above PRVD02) }\end{array}$} & \multirow{2}{*}{$\begin{array}{l}\text { HWM survey } \\
\text { uncertainty } \\
( \pm \mathrm{ft})\end{array}$} \\
\hline & & \multicolumn{2}{|c|}{ Decimal degrees } & & \\
\hline PRSAN23647 & San Juan & 18.4065 & -66.0670 & 25.45 & 0.00 \\
\hline PRSAN23648 & San Juan & 18.4125 & -66.0733 & 16.79 & 0.00 \\
\hline PRSAN23649 & San Juan & 18.4153 & -66.0785 & 15.33 & 0.00 \\
\hline PRSAN23650 & San Juan & 18.4052 & -66.0741 & 20.99 & 0.00 \\
\hline PRSAN23652 & San Juan & 18.4026 & -66.0766 & 23.12 & 0.00 \\
\hline PRUTU23651 & Utuado & 18.2667 & -66.7102 & 439.48 & 0.06 \\
\hline PRUTU23651 & Utuado & 18.2667 & -66.7102 & 439.74 & 0.06 \\
\hline PRARE23653 & Arecibo & 18.4703 & -66.7165 & 15.49 & 0.07 \\
\hline PRARE23654 & Arecibo & 18.4705 & -66.7050 & 12.38 & 0.07 \\
\hline PRCAN23655 & Canovanas & 18.3739 & -65.9159 & 31.68 & 0.05 \\
\hline PRCAN23656 & Canovanas & 18.3720 & -65.9016 & 39.19 & 0.05 \\
\hline PRCOM23658 & Comerio & 18.2240 & -66.2176 & 637.34 & 0.16 \\
\hline PRCAN23659 & Canovanas & 18.3934 & -65.8886 & 24.78 & 0.09 \\
\hline PRMAN23660 & Manati & 18.4283 & -66.5235 & 32.54 & 0.10 \\
\hline PRMAN23661 & Manati & 18.4282 & -66.5056 & 40.82 & 0.10 \\
\hline PRCOM23662 & ComerÃo & 18.2209 & -66.2255 & 648.16 & 0.16 \\
\hline PRMAN23663 & Manati & 18.4273 & -66.4974 & 43.75 & 0.10 \\
\hline PRCOM23664 & Comerio & 18.2181 & -66.2259 & 652.83 & 0.16 \\
\hline PRCOM23665 & Comerio & 18.2355 & -66.2149 & 618.68 & 0.16 \\
\hline PRCOM23666 & Comerio & 18.2496 & -66.2064 & 594.67 & 0.16 \\
\hline PRHUM23667 & Humacao & 18.1637 & -65.7461 & 8.48 & 0.14 \\
\hline PRUTU23668 & Utuado & 18.2736 & -66.7102 & 427.21 & 0.06 \\
\hline PRUTU23669 & Utuado & 18.2694 & -66.7089 & 433.99 & 0.06 \\
\hline PRUTU23670 & Utuado & 18.2725 & -66.7085 & 429.16 & 0.06 \\
\hline PRUTU23671 & Utuado & 18.2829 & -66.7029 & 409.70 & 0.06 \\
\hline PRUTU23672 & Utuado & 18.2604 & -66.6913 & 492.48 & 0.06 \\
\hline PRSAL23673 & Salinas & 17.9775 & -66.3024 & 26.22 & 0.05 \\
\hline PRSAL23674 & Salinas & 17.9761 & -66.3029 & 25.36 & 0.05 \\
\hline PRRIO23676 & Rio Grande & 18.3788 & -65.8290 & 14.98 & 0.08 \\
\hline PRRIO23677 & Rio Grande & 18.3853 & -65.8284 & 12.53 & 0.02 \\
\hline PRRIO23678 & Rio Grande & 18.3853 & -65.8278 & 11.96 & 0.02 \\
\hline PRRIO23679 & Rio Grande & 18.3866 & -65.8284 & 9.21 & 0.02 \\
\hline PRRIO23680 & Rio Grande & 18.3939 & -65.8235 & 8.49 & 0.02 \\
\hline PRHUM23681 & Humacao & 18.1684 & -65.7416 & 9.52 & 0.14 \\
\hline PRRIO23682 & Rio Grande & 18.3938 & -65.8234 & 8.46 & 0.02 \\
\hline PRRIO23684 & Rio Grande & 18.3782 & -65.8292 & 15.15 & 0.08 \\
\hline PRHUM23683 & Humacao & 18.1693 & -65.7467 & 6.97 & 0.14 \\
\hline PRHUM23683 & Humacao & 18.1693 & -65.7467 & 7.21 & 0.14 \\
\hline PRHUM23685 & Humacao & 18.1671 & -65.7522 & 6.19 & 0.14 \\
\hline
\end{tabular}


Table 3. Hurricane Maria high-water marks recorded by the U.S. Geological Survey in Puerto Rico, September 2017.-Continued

[Peak assumed to occur on September 20, 2017. HWM, high-water mark; ft, foot; PRVD02, Puerto Rico Vertical Datum of $2002 ; \pm$, plus or minus]

\begin{tabular}{|c|c|c|c|c|c|}
\hline \multirow{2}{*}{$\begin{array}{c}\text { Site } \\
\text { identification }\end{array}$} & \multirow{2}{*}{ Municipality } & Latitude & Longitude & \multirow{2}{*}{$\begin{array}{c}\text { HWM } \\
\text { surveyed elevation } \\
\text { (ft above PRVD02) }\end{array}$} & \multirow{2}{*}{$\begin{array}{c}\text { HWM survey } \\
\text { uncertainty } \\
( \pm \mathrm{ft})\end{array}$} \\
\hline & & \multicolumn{2}{|c|}{ Decimal degrees } & & \\
\hline PRSAL23686 & Salinas & 17.9867 & -66.2803 & 61.70 & 0.05 \\
\hline PRHUM23685 & Humacao & 18.1671 & -65.7522 & 6.32 & 0.14 \\
\hline PRHUM23687 & Humacao & 18.1622 & -65.7585 & 7.38 & 0.14 \\
\hline PRARE23616 & Arecibo & 18.4667 & -66.7207 & 16.50 & 0.07 \\
\hline PRCAR23688 & Carolina & 18.3755 & -65.9175 & 28.99 & 0.05 \\
\hline PRCAR23688 & Carolina & 18.3755 & -65.9175 & 29.33 & 0.05 \\
\hline PRUTU23689 & Utuado & 18.2550 & -66.6800 & 567.60 & 0.06 \\
\hline PRUTU23690 & Utuado & 18.2662 & -66.6966 & 463.94 & 0.06 \\
\hline PRCAN23691 & Canovanas & 18.3779 & -65.8921 & 43.37 & 0.05 \\
\hline PRCAN23692 & Canovanas & 18.3450 & -65.8961 & 122.75 & 0.09 \\
\hline PRRIO23693 & Rio Grande & 18.3930 & -65.8126 & 8.45 & 0.02 \\
\hline PRRIO23694 & Rio Grande & 18.3786 & -65.8296 & 15.11 & 0.08 \\
\hline PRRIO23695 & Rio Grande & 18.3749 & -65.8239 & 23.65 & 0.08 \\
\hline PRSAL23696 & Salinas & 17.9627 & -66.3005 & 2.09 & 0.05 \\
\hline PRHUM23697 & Humacao & 18.1849 & -65.7081 & 8.75 & 0.14 \\
\hline PRHUM23697 & Humacao & 18.1849 & -65.7081 & 8.98 & 0.14 \\
\hline PRHUM23698 & Humacao & 18.1873 & -65.7109 & 7.73 & 0.14 \\
\hline PRHUM23699 & Humacao & 18.1594 & -65.7641 & 7.92 & 0.14 \\
\hline PRHUM23700 & Humacao & 18.1640 & -65.7615 & 7.81 & 0.14 \\
\hline PRHUM23701 & Humacao & 18.1592 & -65.7589 & 7.88 & 0.14 \\
\hline PRYAU23702 & Yauco & 18.0329 & -66.8440 & 98.80 & 0.60 \\
\hline PRYAU23703 & Yauco & 18.0368 & -66.8446 & 104.43 & 0.60 \\
\hline PRYAU23704 & Yauco & 18.0351 & -66.8460 & 99.31 & 0.60 \\
\hline PRCAN23657 & Canovanas & 18.3386 & -65.8903 & 146.46 & 0.05 \\
\hline PRYAU23706 & Yauco & 18.0388 & -66.8443 & 115.39 & 0.60 \\
\hline PRHUM23708 & Humacao & 18.1879 & -65.7261 & 8.76 & 0.14 \\
\hline PRHUM23709 & Humacao & 18.1893 & -65.7273 & 7.84 & 0.14 \\
\hline PRHUM23710 & Humacao & 18.1879 & -65.7272 & 7.40 & 0.14 \\
\hline PRYAU23711 & Yauco & 18.0385 & -66.8439 & 110.31 & 0.60 \\
\hline PRYAU23712 & Yauco & 18.0337 & -66.8477 & 99.37 & 0.60 \\
\hline PRGUA23713 & Guayama & 17.9760 & -66.0935 & 57.97 & 0.10 \\
\hline PRGUA23714 & Guayama & 17.9744 & -66.1252 & 129.54 & 0.10 \\
\hline PRGUA23715 & Guayama & 17.9771 & -66.1242 & 145.12 & 0.06 \\
\hline PRSAN23716 & Santa Isabel & 17.9552 & -66.4098 & 4.59 & 0.02 \\
\hline PRYAU23717 & Yauco & 18.0318 & -66.8473 & 96.55 & 0.60 \\
\hline PRYAU23718 & Yauco & 18.0299 & -66.8463 & 92.45 & 0.60 \\
\hline PRSAN23719 & Santa Isabel & 17.9564 & -66.4090 & 7.17 & 0.02 \\
\hline PRTOA23720 & Toa Alta & 18.3959 & -66.2529 & 48.11 & 0.08 \\
\hline PRTOA23728 & Toa Alta & 18.3934 & -66.2471 & 52.57 & 0.08 \\
\hline
\end{tabular}


Table 3. Hurricane Maria high-water marks recorded by the U.S. Geological Survey in Puerto Rico, September 2017.-Continued

[Peak assumed to occur on September 20, 2017. HWM, high-water mark; ft, foot; PRVD02, Puerto Rico Vertical Datum of $2002 ; \pm$, plus or minus]

\begin{tabular}{|c|c|c|c|c|c|}
\hline \multirow{2}{*}{$\begin{array}{c}\text { Site } \\
\text { identification }\end{array}$} & \multirow{2}{*}{ Municipality } & Latitude & Longitude & \multirow{2}{*}{$\begin{array}{c}\text { HWM } \\
\text { surveyed elevation } \\
\text { (ft above PRVD02) }\end{array}$} & \multirow{2}{*}{$\begin{array}{l}\text { HWM survey } \\
\text { uncertainty } \\
( \pm \mathrm{ft})\end{array}$} \\
\hline & & \multicolumn{2}{|c|}{ Decimal degrees } & & \\
\hline PRTOA23738 & Toa Alta & 18.3911 & -66.2537 & 49.91 & 0.08 \\
\hline PRCAG23739 & Caguas & 18.1840 & -66.0551 & 359.37 & 0.03 \\
\hline PRCAG23740 & Caguas & 18.2336 & -66.0423 & 210.61 & 0.03 \\
\hline PRVEG23741 & Vega Baja & 18.4620 & -66.3716 & 13.70 & 0.23 \\
\hline PRCAG23742 & Caguas & 18.2230 & -66.0156 & 187.87 & 0.03 \\
\hline PRVEG23743 & Vega Baja & 18.4605 & -66.3569 & 19.42 & 0.23 \\
\hline PRVEG23744 & Vega Baja & 18.4341 & -66.3814 & 42.66 & 0.23 \\
\hline PRTOA23745 & Toa Baja & 18.4109 & -66.2558 & 37.04 & 0.08 \\
\hline PRCAN23746 & Canovanas & 18.3913 & -65.8913 & 24.77 & 0.09 \\
\hline PRCAN23747 & Canovanas & 18.4010 & -65.9109 & 15.97 & 0.01 \\
\hline PRYAU23748 & Yauco & 18.0029 & -66.8340 & 47.88 & 0.60 \\
\hline PRGUA23749 & Guayama & 17.9796 & -66.1232 & 154.36 & 0.06 \\
\hline PRGUA23750 & Guayama & 17.9809 & -66.1226 & 164.15 & 0.10 \\
\hline PRGUA23751 & Guayama & 17.9874 & -66.1182 & 200.49 & 0.10 \\
\hline PRGUA23752 & Guayama & 17.9889 & -66.1168 & 210.55 & 0.10 \\
\hline PRVEG23765 & Vega Baja & 18.4392 & -66.3634 & 35.67 & 0.27 \\
\hline PRVEG23766 & Vega Baja & 18.4344 & -66.3618 & 37.35 & 0.27 \\
\hline PRVEG23767 & Vega Baja & 18.4302 & -66.3563 & 44.01 & 0.23 \\
\hline PRYAU23793 & Yauco & 17.9900 & -66.8400 & 44.58 & 0.60 \\
\hline PRCAR23794 & Carolina & 18.3687 & -65.9540 & 39.49 & 0.09 \\
\hline PRARE23795 & Arecibo & 18.4562 & -66.7241 & 17.07 & 0.07 \\
\hline PRCAR23796 & Carolina & 18.3731 & -65.9459 & 35.55 & 0.09 \\
\hline PRARE23797 & Arecibo & 18.4167 & -66.7077 & 43.48 & 0.07 \\
\hline PRARE23798 & Arecibo & 18.3931 & -66.6823 & 72.80 & 0.07 \\
\hline PRRIN23799 & Rincon & 18.3155 & -67.2246 & 51.43 & 0.06 \\
\hline
\end{tabular}


Table 4. Hurricane Maria peak riverine stage recorded at U.S. Geological Survey long-term monitoring sites in Puerto Rico, September 2017.

[Dates shown as month, day, year. Time shown in 24-hour format. ft, foot; UTC, Coordinated Universal Time; blw, below; nr, near; abv, above; hwy, highway; *, provisional; >, greater than; E, estimated; PR, Puerto Rico]

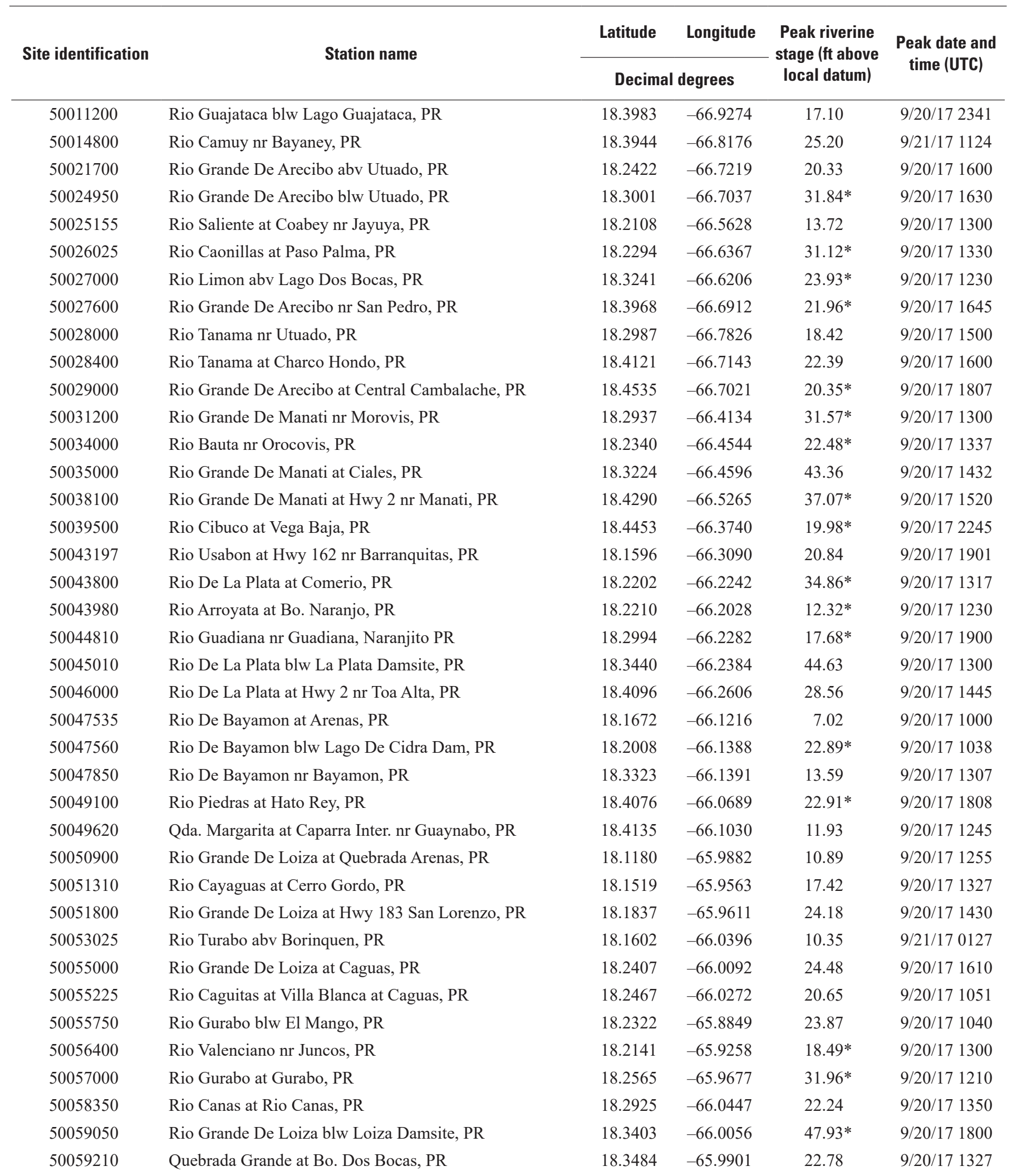


Table 4. Hurricane Maria peak riverine stage recorded at U.S. Geological Survey long-term monitoring sites in Puerto Rico, September 2017.-Continued

[Dates shown as month, day, year. Time shown in 24-hour format. ft, foot; UTC, Coordinated Universal Time; blw, below; nr, near; abv, above; hwy, highway; *, provisional; >, greater than; E, estimated; PR, Puerto Rico]

\begin{tabular}{|c|c|c|c|c|c|}
\hline \multirow{2}{*}{ Site identification } & \multirow{2}{*}{ Station name } & Latitude & Longitude & \multirow{2}{*}{$\begin{array}{l}\text { Peak riverine } \\
\text { stage (ft above } \\
\text { local datum) }\end{array}$} & \multirow{2}{*}{$\begin{array}{l}\text { Peak date and } \\
\text { time (UTC) }\end{array}$} \\
\hline & & \multicolumn{2}{|c|}{ Decimal degrees } & & \\
\hline 50061800 & Rio Canovanas nr Campo Rico, PR & 18.3165 & -65.8885 & $17.33^{*}$ & $9 / 20 / 170945$ \\
\hline 50063800 & Rio Espiritu Santo nr Rio Grande, PR & 18.3579 & -65.8136 & 17.24 & 9/20/17 1146 \\
\hline 50064200 & Rio Grande $\mathrm{nr}$ El Verde, PR & 18.3434 & -65.8416 & 18.48 & 9/20/17 1145 \\
\hline 50065500 & Rio Mameyes nr Sabana, PR & 18.3268 & -65.7504 & 12.10 & $9 / 20 / 171300$ \\
\hline 50067000 & Rio Sabana at Sabana, PR & 18.3290 & -65.7306 & 16.47 & $9 / 20 / 171120$ \\
\hline 50070900 & Rio Fajardo at Paraiso nr Fajardo, PR & 18.2808 & -65.7007 & 12.52 & 9/20/17 0922 \\
\hline 50071000 & Rio Fajardo nr Fajardo, PR & 18.2970 & -65.6934 & 13.90 & $9 / 20 / 171205$ \\
\hline 50075000 & Rio Icacos nr Naguabo, PR & 18.2752 & -65.7854 & 8.35 & $9 / 20 / 171150$ \\
\hline 50081000 & Rio Humacao at Las Piedras, PR & 18.1721 & -65.8690 & 6.63 & $9 / 20 / 171405$ \\
\hline 50083500 & Rio Guayanes nr Yabucoa, PR & 18.0569 & -65.9007 & 24.79 & $9 / 20 / 171500$ \\
\hline 50085100 & Rio Guayanes at Central Roig, PR & 18.0644 & -65.8738 & 27.29 & $9 / 20 / 171455$ \\
\hline 50090500 & Rio Maunabo at Lizas, PR & 18.0252 & -65.9391 & 11.86 & 9/20/17 1301 \\
\hline 50092000 & Rio Grande De Patillas nr Patillas, PR & 18.0322 & -66.0321 & 16.38 & $9 / 20 / 171203$ \\
\hline 50093000 & Rio Marin nr Patillas, PR & 18.0358 & -66.0093 & 11.50 & $9 / 20 / 171015$ \\
\hline 50093120 & Rio Grande De Patillas blw Lago Patillas, PR & 18.0144 & -66.0232 & 16.94 & $9 / 20 / 171200$ \\
\hline 50100200 & Rio Lapa nr Rabo Del Buey, PR & 18.0577 & -66.2407 & 15.37 & 9/20/17 1155 \\
\hline 50100450 & Rio Majada at La Plena, PR & 18.0427 & -66.2071 & $16.62^{*}$ & $9 / 20 / 171155$ \\
\hline 50110650 & Rio Jacaguas abv Lago Guayabal & 18.1146 & -66.5043 & $17.19^{*}$ & $9 / 20 / 171242$ \\
\hline 50110900 & Rio Toa Vaca abv Lago Toa Vaca, PR & 18.1245 & -66.4574 & $14.36^{*}$ & $9 / 20 / 171600$ \\
\hline 50112500 & Rio Inabon at Real Abajo, PR & 18.0845 & -66.5627 & $>20.76$ & 9/20/17 1331 \\
\hline 50113800 & Rio Cerrillos abv Lago Cerrillos nr Ponce, PR & 18.1148 & -66.6045 & 15.55 & $9 / 20 / 171222$ \\
\hline 50114000 & Rio Cerrillos blw Lago Cerrillos nr Ponce, PR & 18.0709 & -66.5810 & 7.53 & $9 / 20 / 171053$ \\
\hline 50114900 & Rio Portugues nr Tibes, PR & 18.0979 & -66.6425 & 14.04 & $9 / 20 / 171357$ \\
\hline 50115240 & Rio Portugues at Parque Ceremonial Tibes nr Ponce & 18.0416 & -66.6211 & 8.20 & $9 / 20 / 171315$ \\
\hline 50124200 & Rio Guayanilla Near Guayanilla, PR & 18.0423 & -66.7978 & 21.40 & $9 / 20 / 171510$ \\
\hline 50126150 & Rio Yauco abv Diversion Monserrate nr Yauco, PR & 18.0472 & -66.8415 & $16.93 \mathrm{E}$ & $9 / 20 / 171210$ \\
\hline 50129254 & Rio Loco at Las Latas nr La Joya nr Guanica, PR & 18.0072 & -66.8763 & $19.08^{*}$ & $9 / 20 / 171750$ \\
\hline 50136400 & Rio Rosario nr Hormigueros, PR & 18.1581 & -67.0854 & 19.50 & 9/20/17 1717 \\
\hline 50138000 & Rio Guanajibo nr Hormigueros, PR & 18.1407 & -67.1484 & 28.59 & $9 / 21 / 170110$ \\
\hline 50144000 & Rio Grande De Anasco nr San Sebastian, PR & 18.2822 & -67.0506 & $35.85^{*}$ & 9/20/17 1904 \\
\hline 50148890 & Rio Culebrinas at Margarita Damsite nr Aguada, PR & 18.3925 & -67.1508 & $21.99 *$ & $9 / 20 / 172130$ \\
\hline
\end{tabular}


Table 5. Hurricane Maria peak storm-tide data recorded at National Oceanic and Atmospheric Administration real-time tide-gages in Puerto Rico and the U.S. Virgin Islands, September 2017.

[Dates shown as month, day, year. Time shown in 24-hour format. PRVD02, Puerto Rico Vertical Datum of 2002; VIVD09, Virgin Islands Vertical Datum of 2009; UTC, Coordinated Universal Time; ft, foot; P.R., Puerto Rico; V.I., Virgin Islands; NOAA, National Oceanic and Atmospheric Administration; \pm , plus or minus]

\begin{tabular}{|c|c|c|c|c|c|c|c|}
\hline \multirow{2}{*}{$\begin{array}{c}\text { Site } \\
\text { identification }\end{array}$} & \multirow{2}{*}{$\begin{array}{c}\text { U.S. } \\
\text { Territory }\end{array}$} & \multirow{2}{*}{ Municipality } & Latitude & Longitude & \multirow{2}{*}{$\begin{array}{c}\text { Peak } \\
\text { storm-tide } \\
\text { elevation } \\
\text { (ft) }\end{array}$} & \multirow{2}{*}{$\begin{array}{l}\text { Peak storm-tide } \\
\text { date and time } \\
\text { (UTC) }\end{array}$} & \multirow{2}{*}{$\begin{array}{c}\text { Vertical } \\
\text { datum }\end{array}$} \\
\hline & & & \multicolumn{2}{|c|}{ Decimal degrees } & & & \\
\hline 9751381 & V.I. & St. John & 18.3169 & -64.7181 & 1.84 & 9/21/20170718 & VIVD09 \\
\hline 9752619 & P.R. & Vieques Island & 18.1503 & -65.4350 & $2.63 \pm 0.02$ & 9/21/20170800 & PRVD02 \\
\hline 9754228 & P.R. & Yabucoa & 18.0508 & -65.8333 & $5.66 \pm 0.02$ & 9/20/2017 1118 & PRVD02 \\
\hline 9755371 & P.R. & San Juan & 18.4517 & -66.1167 & 3.10 & 9/20/2017 1200 & PRVD02 \\
\hline 9757809 & P.R. & Arecibo & 18.4689 & -66.7003 & $2.63 \pm 0.02$ & 9/20/20171418 & PRVD02 \\
\hline 9759938 & P.R. & Mona Island & 18.0844 & -67.9342 & 2.76 & 9/21/20170148 & PRVD02 \\
\hline
\end{tabular}

Table 6. Precipitation measured in the U.S. Virgin Islands and Puerto Rico, September 18-22, 2017.

[in., inch; USVI, U.S. Virgin Islands; PR, Puerto Rico; hwy, highway]

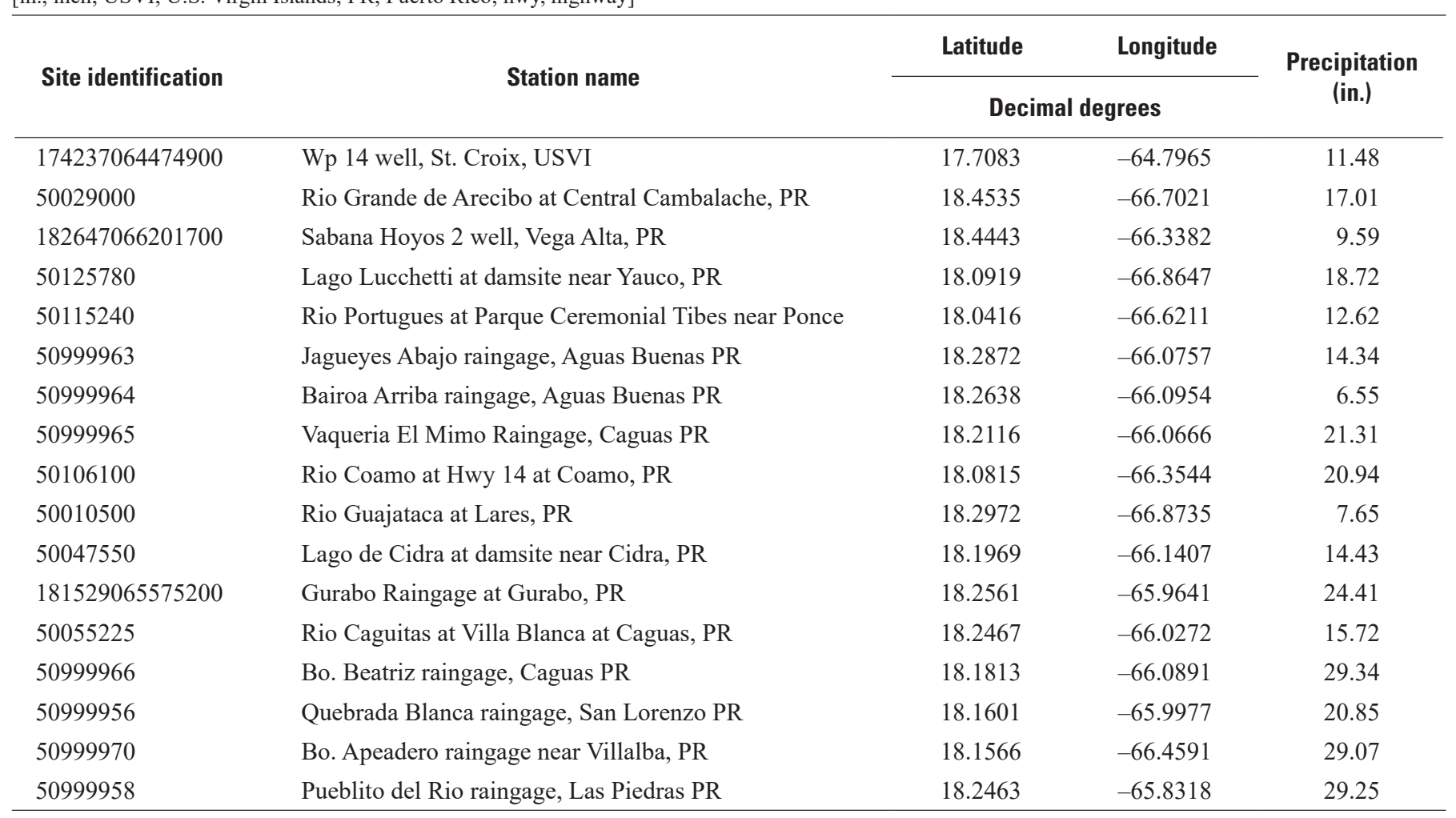




\section{Conclusion}

Hurricane Maria made landfall south of Yabucoa Harbor, Puerto Rico, as a category 4 hurricane with maximum sustained winds of 155 miles per hour on September 20, 2017. The hurricane devastated much of the U.S. Virgin Islands and Puerto Rico. The U.S. Geological Survey (USGS), in cooperation with the Federal Emergency Management Agency, installed a temporary monitoring network of 13 water-level and barometric pressure sensors along the coast of Puerto Rico prior to the storm. In addition to the temporary sensors, the USGS maintains 99 permanent real-time streamgages and 36 real-time precipitation stations in Puerto Rico. The real-time data, updated hourly during and after the hurricane, are displayed in the USGS Flood Event Viewer (https://stn.wim.usgs.gov/FEV/\#MariaSeptember2017) and in the USGS National Water Information System. The USGS measured 181 coastal and riverine high-water marks throughout Puerto Rico after the storm. Water elevations are referenced to the Puerto Rico Vertical Datum of 2002 (PRVD02) and local datums in Puerto Rico and to the U.S. Virgin Islands Vertical Datum of 2009 (VIVD09) in the U.S. Virgin Islands.

The data from Hurricane Maria storm-tide network are available in tab-delimited, ASCII format and Network Common Data Form (NetCDF) format by site for each sensor through the USGS Flood Event Viewer. The peak storm tide was recorded at 11 water-level sensors, and the peak riverine stage was recorded at 70 permanent stations. Peak storm-tide data also were compiled for National Oceanic and Atmospheric Administration tide-gages, which have instrumentation to measure coastal water levels located in Puerto Rico and the U.S. Virgin Islands. Precipitation was recorded at 17 permanent stations.

\section{References Cited}

Autoridad de Energía Eléctrica [Puerto Rico Electric Power Authority], 2018, Se completan los trabajos de energización del último cliente*** [The energization work of the last client is completed $* * *]$ : Twitter website, [@AEEONLINE], August 14, 2018, accessed April 22, 2019, at https://twitter.com/AEEONLINE/ status/1029435301176004608.

Cangialosi, J.P., Latto, A.S., and Berg, R., 2018, Hurricane Irma 30 August-12 September 2017: National Hurricane Center Tropical Cyclone Report, accessed December 19, 2018, at https:/www.nhc.noaa.gov/data/tcr/ AL112017_Irma.pdf.
Davidian, J., 1976, Computation of water-surface profiles in open channels: U.S. Geological Survey Techniques of Water Resources Investigations, book 3, chap. A15, 57 p., accessed May 29, 2019, at https://pubs.usgs.gov/twri/twri3a15/pdf/TWRI_3-A15.pdf.

Federal Aviation Administration, 2017, Commercial airline service resumes in San Juan: FAA Home, News, News \& Updates, accessed March 16, 2018, at https:/www.faa.gov/ news/updates/?newsId $=88805$.

Frantz, E.R., Byrne, M.J., Sr., Caldwell, A.W., and Harden, S.L., 2017, Monitoring storm tide and flooding from Hurricane Matthew along the Atlantic Coast of the United States, October 2016: U.S. Geological Survey Open-File Report 2017-1122, 37 p., accessed April 1, 2019, at https://doi.org/10.3133/ofr20171122.

Koenig, T.A., Bruce, J.L., O’Connor, J.E., McGee, B.D., Holmes, R.R., Jr., Hollins, R., Forbes, B.T., Kohn, M.S., Schellekens, M.F., Martin, Z.W., and Peppler, M.C., 2016, Identifying and preserving high-water mark data: U.S. Geological Survey Techniques and Methods, book 3, chap. A24, 47 p., accessed April 1, 2019, at https://doi. org/10.3133/tm3A24.

Matthai, H.F., 1967, Measurement of peak discharge at width contractions by indirect methods: U.S. Geological Survey Techniques of Water-Resources Investigations, book 3, chap. A4, 44 p, accessed April 1, 2019, at https://pubs.usgs. gov/twri/twri3-a4/.

McCallum, B.E., Painter, J.A., and Frantz, E.R., 2012, Monitoring storm tide and flooding from Hurricane Irene along the Atlantic coast of the United States, August 2011: U.S. Geological Survey Open-File Report 2012-1022, accessed April 1, 2019, at http://pubs.usgs.gov/ of/2012/1022/.

McGee, B.D., Goree, B.B., Tollett, R.W., Woodward, B.K., and Kress, W.H., 2006, Hurricane Rita surge data, southwestern Louisiana and southeastern Texas, September to November 2005: U.S. Geological Survey Data Series 220, accessed April 1, 2019, at http://pubs.usgs.gov/ ds/2006/220/.

National Geodetic Survey [NGS], 2017, Notice: NGS update, May 16, 2017, technical details for GEOID12/12A/12B:

The NGS Geoid Page, accessed February 27, 2018, at https://www.ngs.noaa.gov/GEOID/GEOID12B/ GEOID12B_TD.shtml.

National Hurricane Center, 2012, The Saffir-Simpson Hurricane Wind Scale: National Oceanic and Atmospheric Administration, National Hurricane Center website, accessed December 1, 2016, at http://www.nhc.noaa.gov/ aboutsshws.php. 
National Oceanic and Atmospheric Administration [NOAA], 2017, Tides \& currents: National Oceanic and Atmospheric Administration web page, accessed February 27, 2018, at https://tidesandcurrents.noaa.gov/stations.html?type $=$ Water + Levels.

Pasch, R.J., Penny, A.B., and Berg, R., 2018, Hurricane Maria 16-30 September 2017, National Hurricane Center Tropical Cyclone Report, April 10, 2018, accessed December 19, 2018, at https:/www.nhc.noaa.gov/data/tcr/ AL152017_Maria.pdf.

Rydlund, P.H., Jr., and Densmore, B.K., 2012, Methods of practice and guidelines for using survey-grade Global Navigation Satellite Systems (GNSS) to establish vertical datum in the United States Geological Survey: U.S. Geological Survey Techniques and Methods, book 11, chap. D1, 102 p. with appendixes, accessed April 1, 2019, at https://pubs.usgs.gov/tm/11d1/tm11-D1.pdf.

U.S. Census Bureau, 2010, 2010 Census island areasU.S. Virgin Islands: United States Census Bureau web page, accessed March 13, 2018, at https:/www.census. gov/2010census/news/press-kits/island-areas/island-areas.html.
U.S. Census Bureau, 2017, Population estimate as of July 1, 2017: U.S. Census Bureau web page, accessed March 13, 2018, at https://factfinder.census.gov/faces/nav/ jsf/pages/community_facts.xhtml?src=bkmk.

U.S. Department of Energy, 2017, Hurricane Irma \& Hurricane Harvey-Event report (update no. 23): U.S. Department of Energy report, accessed December 20, 2018, at https://www.energy.gov/sites/ prod/files/2017/10/f37/Hurricanes\%20Irma\%20and\%20 Harvey\%20Event \%20Summary\%20\%2323.pdf.

U.S. Department of Energy, 2018, Hurricanes Maria \& IrmaJanuary 31 event summary (report no. 89): U.S. Department of Energy report, accessed December 20, 2018, at https://www.energy.gov/sites/prod/files/2018/01/f47/ Hurricanes\%20Maria\%20and\%20Irma\%20Event $\% 20$ Summary\%20January\%2031\%2C\%202018.pdf.

U.S. Geological Survey, 2016, USGS water data for the Nation: U.S. Geological Survey National Water Information System web interface, accessed February 6, 2019, at https://waterdata.usgs.gov/nwis. 
For more information about this publication, contact

Director, Caribbean-Florida Water Science Center

U.S. Geological Survey

4446 Pet Lane, Suite 108

Lutz, FL 33559

(813) 498-5000

For additional information visit

https://www2.usgs.gov/water/caribbeanflorida/index.html

Publishing support provided by

Lafayette Publishing Service Center 

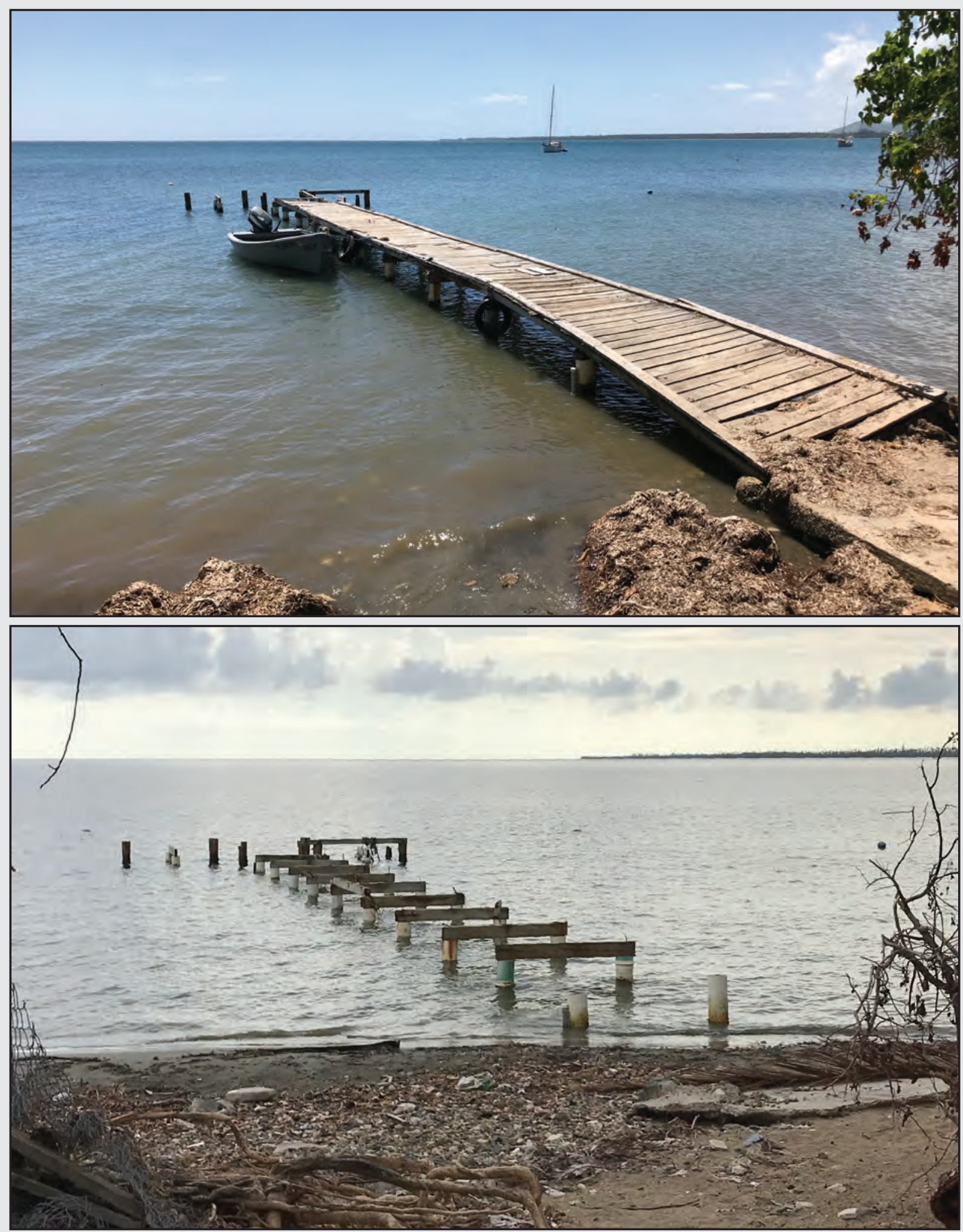

ISSN 0196-1497 (print) 